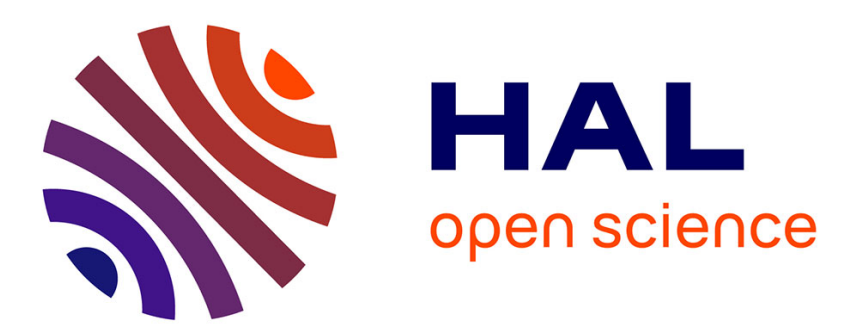

\title{
Agressions indirectes des enseignants par les parents d'élèves : deux exemples d'interventions cognitivo-comportementales dans une école primaire grecque
}

\author{
Sylvie Heitzmann-Kamarinos, Céline Clément
}

\section{To cite this version:}

Sylvie Heitzmann-Kamarinos, Céline Clément. Agressions indirectes des enseignants par les parents d'élèves: deux exemples d'interventions cognitivo-comportementales dans une école primaire grecque. Journal de Thérapie Comportementale et Cognitive, 2011, 21, pp.37-42. hal-00751389

\author{
HAL Id: hal-00751389 \\ https://hal.science/hal-00751389
}

Submitted on 14 Nov 2012

HAL is a multi-disciplinary open access archive for the deposit and dissemination of scientific research documents, whether they are published or not. The documents may come from teaching and research institutions in France or abroad, or from public or private research centers.
L'archive ouverte pluridisciplinaire HAL, est destinée au dépôt et à la diffusion de documents scientifiques de niveau recherche, publiés ou non, émanant des établissements d'enseignement et de recherche français ou étrangers, des laboratoires publics ou privés. 
Agressions indirectes des enseignants par les parents d'élèves : deux exemples d'interventions cognitivo-comportementales dans une école primaire grecque

Cognitive and behavioral approach in the resolution of indirect aggressions of teachers by parents: two single cases in a Greek school

Heitzmann-Kamarinos, Sylvie (a), Clément, Céline (b)

a) Psychologue, doctorante, Université de Strasbourg, France

b) Professeur, Laboratoire Interuniversitaire des Sciences de l'Éducation et de la Communication LISEC - EA 2310 et IUFM d'Alsace, Université de Strasbourg, France.

Auteur correspondant : Sylvie Heitzmann-Kamarinos

sylkamharis@yahoo.com 


\title{
Résumé
}

L'agression indirecte des enseignants par les parents d'élèves est un problème fréquent, aux répercussions psychologiques importantes et face auquel les enseignants se sentent souvent démunis. Les interventions comportementales et cognitives basées sur l'analyse fonctionnelle des comportements perturbateurs sont une réponse possible. Cet article présente deux interventions de ce type dans une école primaire grecque. L'efficacité des modèles d'analyse fonctionnelle est démontrée. Les techniques employées sont détaillées. Les limites de telles interventions dans le système scolaire grec sont analysées. Enfin, l'intérêt de former les enseignants aux techniques comportementales et cognitives est discuté.

Mots clés : agression indirecte, analyse fonctionnelle, plan d'intervention, relations enseignants-parents

\begin{abstract}
Teachers indirect aggression by pupils parents is a frequent problem, with important psychological consequences and teachers often don't know how to deal with. Cognitive and behavioral interventions based on functional analysis of problembehaviors are a possible response. This paper shows the results of two interventions of this type in a primary school in Greece. The efficacy of functional analysis models is demonstrated. The technics used are detailed. The limits of these interventions in the Greek school system are analyzed. Finally, the interest to train teachers to cognitive and behavioral technics is discussed.
\end{abstract}

Key words: indirect aggression, functional analysis, intervention, teacher-parent relationship 
La violence à l'école est un fait préoccupant en constante augmentation [1], pour laquelle les psychologues sont de plus en plus souvent sollicités [2]. Si elle concerne le plus souvent les pairs entre eux, elle touche aussi les enseignants. Ces derniers font partie des nouvelles cibles de la violence au travail [3]. Les conflits avec les parents d'élèves sont une véritable préoccupation pour les enseignants [4], leur origine étant souvent la contestation d'une décision concernant l'enfant, d'une note ou d'une punition [4].

En France, dans deux tiers des cas de violences subis, les enseignants mettent en cause les parents d'élèves [4]. La fréquence de ces agressions n'est pas connue en Grèce, mais les médias se font fréquemment l'écho d'agressions de parents d'élèves envers les enseignants.

Cette violence peut prendre la forme d'une simple incivilité ou d'une agression verbale ou physique, avec volonté de blesser ou de détruire [3]. Si elle est souvent directe, elle peut également être indirecte, relationnelle et inclut alors le fait d'encourager les autres à ne pas aimer quelqu'un, à l'éviter, à répandre des rumeurs le concernant [5]. C'est dans ce cas une forme de manipulation sociale où la cible n'est pas agressée directement mais de façon détournée, ce qui permet à l'agresseur d'éviter la contre-attaque [6]. Cette forme d'agressivité est fréquemment utilisée par les femmes, étant peu couteuse psychologiquement et socialement pour l'agresseur, mais elle est aussi utilisée par les hommes [7].

En revanche, elle a des répercussions psychologiques importantes pour la victime, entrainant dépression et troubles anxieux, jusqu'à des états de stress post-traumatique dans les cas les plus graves [3] et est un facteur favorisant de l'épuisement professionnel [8]. Ces éléments justifient une intervention en urgence pour la résolution du problème.

Les interventions cognitivo-comportementales offrent une palette de techniques bien adaptées au milieu scolaire [9] car tenant compte de l'intrication de tous les facteurs environnementaux. Celles-ci découlent de l'analyse fonctionnelle qui permet d'articuler le comportement-problème avec ses antécédents, ses facteurs déclenchant et ses conséquences, tout en repérant les facteurs de maintien du comportementproblème [10] pour mettre en place un plan d'intervention qui se doit d'être économique et efficace [11].

L'analyse comportementale peut se faire selon différents modèles, dont le modèle antécédents-stimulus-comportement-conséquences inspiré des théories de Skinner 
[10] et le modèle SECCA [12] utilisé dans les deux cas présentés ci-après et dont l'intérêt dans le cas d'une intervention en urgence sera discuté.

Cet article présente l'intervention cognitivo-comportementale faite dans une école primaire grecque pour résoudre une agression indirecte de deux enseignants par un parent d'élèves. Les analyses fonctionnelles sont résumées afin de mettre en évidence les facteurs de maintien de l'agression et les interventions qui en découlent. Les étapes des interventions avec les différentes techniques travaillées avec les deux enseignants et les parents agresseurs sont discutées ainsi que les limites d'une telle intervention. Des actions de prévention de telles agressions sont proposées.

\section{Méthode}

\section{Participants}

L'école primaire d'Athènes où se passent les évènements décrits compte 348 élèves, répartis en 8 classes de la $1^{\text {ère }}$ à la $6^{\text {ème }}$ année (celle-ci correspond à la classe de 6ème en France). Les parents d'élèves sont donc au nombre de 696 environ, en majorité grecs (moins de 10\% d'enfants d'origine étrangère).

Les enseignants victimes sont ceux des classes de 3ème année et de 6ème année. C'est la deuxième année consécutive qu'ils suivent les élèves de ces classes. Ils sont tous les deux titulaires et ont une expérience professionnelle de 30 ans pour l'enseignante de $3^{\text {ème }}$ année et de 6 ans pour l'enseignant de $6^{\text {ème }}$ année. Les parents présentant des comportements d'agression indirecte sont respectivement : 1) M. H. père de Nikos, élève de $3^{\text {ème }}$ année, 56 ans, exerçant dans un cadre libéral une profession de la santé (3 enfants scolarisés à l'école) et 2) Mme M. mère de Paúlos, élève de $6^{\text {ème }}$ année, 34 ans, sans emploi, isolée et ayant des antécédents de dépression et des problèmes de santé ; elle a deux enfants scolarisés à l'école.

\section{Procédure}

La procédure a été mise en place par une psychologue cognitivo-comportementaliste, intervenante bénévole, travaillant depuis trois ans en collaboration étroite avec l'équipe pédagogique, sur la demande du directeur de l'école.

Afin de repérer les comportements problèmes, leurs causes éventuelles et leurs conséquences, des entretiens semi-directifs individuels ont été conduits avant l'intervention, avec les enseignants victimes ( 2 entretiens de 2 heures) et des parents concernés ( 1 entretien de 2 heures). Les parents ayant participé aux entretiens sont Mme M., mère de Paúlos, élève de 6ème année, et Mme H., épouse de M. H qui a 
refusé de participer aux entretiens, mère de Nikos, élève de $3^{\text {ème }}$ année. Les enseignants et les parents ont été informés de la confidentialité des propos qu'ils tiendraient dans le cadre de ces entretiens individuels.

Le problème repéré est l'agressivité indirecte des parents par rapport à l'enseignant. Une analyse comportementale a été effectuée ensuite, dans les deux cas, sur le modèle antécédents-stimulus-comportement-conséquences, inspiré du modèle SORC, afin de mettre en évidence les facteurs favorisant, les facteurs déclenchant et les facteurs de maintien du comportement-problème, ainsi que l'enchainement des différents comportements d'agression entre enseignants et parents. Une grille SECCA [12] a été utilisée en deuxième lieu pour l'analyse du comportement individuel des enseignants, afin de repérer les éléments émotionnels et cognitifs en cause dans le maintien du comportement agressif.

Le plan expérimental retenu afin de pouvoir mesurer les effets de l'intervention, est un plan ABA', A étant la ligne de base, B étant la phase d'intervention et A' étant la phase de suivi.

La ligne de base correspondant à la mesure de la fréquence des comportements agressifs est effectuée par observation directe (possible dans un cas) et par recueil d'informations auprès des parents et des enseignants.

L'intervention auprès des enseignants ( 2 fois 2 heures) a été programmée, tenant compte des facteurs déclenchant et de maintien repérés par les analyses fonctionnelles.

Une phase de suivi tout au long de l'année scolaire par entretien individuel non directif de 30 minutes à 1 mois, à 3 mois et 6 mois avec les enseignants et les parents agresseurs a permis de vérifier l'absence de reprise de l'agression et le maintien des résultats.

\section{Résultats}

Analyses fonctionnelles

L'analyse comportementale a mis en évidence l'existence d'un comportement agressif de la part des deux enseignants : 1) en classe (cris fréquents, gestes agressifs, paroles agressives envers les élèves face à un comportement jugé non respectueux ou perturbateur) ; 2) vis-à-vis des parents en réunion de parents d'élèves (peu de temps de parole laissé aux parents, beaucoup de critiques directes aux parents rendus responsables des problèmes des élèves). Du coté des agresseurs, il a été repéré un 
mécontentement depuis un an et des critiques répétées aux autres parents de la classe concernant le comportement de chaque enseignant et de ses compétences pédagogiques.

Dans ce contexte, le facteur déclenchant l'agression a été un désaccord de Mme M. par rapport à une évaluation de l'enseignant de $6^{\text {ème }}$ année et un désaccord de M. H. par rapport à une méthode d'enseignement de l'enseignante de $3^{\text {ème }}$ année.

Le comportement agressif, comportement-problème, s'est traduit 1) par des accusations répétées de Mme $\mathrm{M}$. concernant le comportement dangereux et nocif de l'enseignant aux autres parents d'élèves devant l'école à l'heure de la rentrée en classe des élèves ou dans la cour de l'école en attendant leur sortie et 2) par des attaques verbales répétées (injures, dénigrement systématique) de M. H. en famille et devant l'élève, des critiques sur la pédagogie de l'enseignante avec incitation de la part du père à ne pas suivre les consignes de l'enseignante.

La ligne de base de la fréquence des comportements d'agression indirecte avant intervention montrait 1) dans le cas de l'agression indirecte de l'enseignant de $6^{\text {ème }}$ année, une discussion de 10 à 15 min de Mme M. avec les parents rencontrés devant l'école, tous les matins, pendant 10 jours, associés à des appels téléphoniques de plus d'une heure à 5 parents; 2) dans le cas de M. H. agressant indirectement l'enseignante de $3^{\text {ème }}$ année, c'est Mme H. mère de Nikos, et seule interlocutrice qui révèle à la psychologue en entretien, l'existence de critiques quotidiennes sur le travail et les méthodes de l'enseignante (le père faisant les devoirs avec l'enfant, la mère étant d'origine étrangère).

Les conséquences des comportements agressifs ont été importantes dans les deux cas pour les enseignants et pour les élèves.

Dans le cas de Mme M. accusant l'enseignant de $6^{\text {ème }}$ année, l'agressivité indirecte a produit en retour : 1) une augmentation de l'agressivité de l'enseignant en classe (perte de contrôle devant les élèves, augmentation des cris) qui a renforcé à son tour l'agression de la mère, un repli sur soi avec un évitement de l'enseignant et un refus opposé à la psychologue de demander l'arbitrage du directeur de l'école 2) une augmentation de l'agressivité des élèves entre eux avec une augmentation des conflits entre les élèves dont les parents sont «pour» et ceux dont les parents sont «contre» l'enseignant ; 3) une division des parents en groupes « pour » et « contre ».

Dans le cas de M. H. remettant en cause la compétence professionnelle de l'enseignante de $3^{\text {ème }}$ année, son agressivité indirecte a provoqué : 1) une agressivité 
non verbale avec une remise en cause de l'autorité de l'enseignante par l'élève (refus de suivre ses conseils en mathématique) aggravant des difficultés scolaires préexistantes ; 2) une augmentation de l'agressivité de l'enseignante face à son élève (se traduisant par des critiques à Nikos et des punitions répétées) et à sa mère, seule interlocutrice (avec accusations ouvertes d'incompétences parentales et jugement personnel sur le fonctionnement familial et les choix parentaux).

Les stratégies des enseignants pour faire face à l'agression ont été différentes. L'enseignant de $6^{\text {ème }}$ année a décidé de faire face seul, en organisant une réunion des parents en urgence. L'enseignante de $3^{\text {ème }}$ année a demandé un entretien avec M. H. par l'intermédiaire de Mme H. (cet entretien n'a jamais eu lieu, M. H. ayant indiqué avoir des horaires incompatibles avec ceux de l'enseignante et l'enseignante n'acceptant pas de moduler ses heures d'entretien en fonction de M. H.).

L'arbitrage du directeur de l'école n'a été sollicité ni par l'un ni par l'autre enseignant.

Les facteurs de maintien de l'agressivité ont été identifiés.

Dans les deux cas on repère deux facteurs de maintien possibles. Le premier est l'absence d'intervention du directeur pouvant jouer le rôle d'arbitre et de médiateur. Il a été informé tardivement par Mme H., alors que le comportement de l'enseignante et le comportement agressif du père avaient déjà eu des conséquences graves sur le fonctionnement de Nikos à l'école, avec une chute des résultats scolaires et l'apparition d'un comportement opposant en classe. Dans le cas de Mme M., il intervient de son propre chef suite à des rumeurs. Le deuxième facteur de maintien possible est l'évitement de la confrontation de la part des deux parents agresseurs, opposant un refus au psychologue et au directeur à la proposition faite par téléphone de rencontrer les enseignants afin de régler leurs différends en face à face (Mme M. ayant peur des retombées que cet entrevue pourrait avoir sur son fils, l'enseignant pouvant chercher à « se venger »; M. H. considérant que c'était une perte de temps). Dans le cas de Mme M., isolée avant les faits, un $3^{\text {ème }}$ facteur de maintien est repéré ; il s'agit de l'attention sociale dont elle bénéficie puisqu'elle se trouve au centre de l'attention des parents d'élèves et meneuse du groupe « contre » l'enseignant.

Les grilles SECCA ont mis en évidence des facteurs personnels de maintien de l'agression : 1) la difficulté à contrôler ses émotions et notamment sa colère pour l'enseignant de $6^{\text {ème }}$ année et sa tendance au repli sur soi avec des croyances 
dysfonctionnelles («Je dois toujours faire face seul»);2) pour l'enseignante, une tendance à la dépression avec des pensées d'autoaccusation («C'est de ma faute. Je provoque ce genre de comportement ») et au niveau émotionnel, une tristesse et des pensées anticipatoires négatives par rapport à l'élève («Qu'est ce que je vais encore rencontrer aujourd'hui comme problème avec cet élève ?») sont associées à une anxiété anticipatoire perturbant son enseignement et ses interactions avec l'élève, fils de l'agresseur.

\section{Plans d'intervention}

Face aux conséquences importantes de l'agression sur les enseignants et sur les élèves, un plan d'intervention a été décidé à chaque fois en urgence par le directeur de l'établissement en collaboration avec la psychologue sollicitée comme conseiller comportemental [2], et ce dès lors qu'il a été informé de la situation.

Les objectifs de ces plans sont 1) l'arrêt immédiat de l'agression indirecte (arrêt du colportage des rumeurs et de la demande de renvoi de l'enseignant de $6^{\text {ème }}$ année ; arrêt de la critique systématique et de l'agressivité verbale indirecte de la part du père pour l'enseignante de $3^{\text {ème }}$ année ; 2) l'arrêt de l'évitement pour la reprise d'une communication directe et non agressive entre le parent agresseur et l'enseignant, 3) le rétablissement du directeur dans son rôle de tiers entre l'enseignant et le parent agresseur ; 4) la reprise des cours en tout sérénité dans le cas de l'enseignant de $6^{\text {ème }}$ année et la reprise d'interactions positives entre l'enseignante de $3^{\text {ème }}$ année et son élève pour une poursuite des apprentissages et l'acceptation de son aide par l'élève.

Les interventions consistent en une prise en charge de chaque enseignant victime.

Les techniques comportementales et cognitives utilisées auprès des enseignants sont :

- des techniques d'affirmation de soi [13,14], l'accent étant mis sur a) la capacité à écouter l'autre, sur la reconnaissance du point de vue de l'interlocuteur (reconnaitre les compétences du parent agresseur en tant que personne ayant une connaissance intime de son enfant, reconnaitre les difficultés des parents avant de les accuser de «mal éduquer » leurs enfants), b) l'expression des émotions (exprimer la colère ressentie par rapport aux accusations de maltraitance de la mère d'élève ou la tristesse ressentie par rapport aux critiques du parent sur les méthodes pédagogiques), c) le langage non verbal (contrôle de l'expression faciale et du ton de la voix pour maitriser sa colère), d) la compétence à recevoir et à formuler une critique négative 
envers un élève ou un parent d'élèves (recevoir une critique par rapport à ses compétences pédagogiques en demandant des exemples concrets ou exemples précis de situations en réunions où l'enseignant a été jugé agressif avec les parents) et d) la capacité de faire des compliments (l'utilisation des renforcements positifs en réunion de parents, avant de faire des critiques ou d'aborder les problèmes avec les élèves en classe). Ces techniques sont apprises par jeux de rôle [15] à raison de deux séances de travail avec chaque enseignant sur une semaine, afin de préparer la confrontation avec les parents d'élèves agresseurs.

- l'apprentissage du contrôle émotionnel : contrôle de la colère [16,17] et de l'anxiété $[18,19]$ par contrôle respiratoire et relaxation musculaire, avant et pendant la confrontation avec les parents.

- des techniques cognitives avec l'apprentissage du modèle dit «modèle de Beck» [20] : a) le repérage des pensées anticipatoires anxiogènes par rapport aux parents ou aux élèves et leurs conséquences sur le comportement (par exemple l'agressivité qui en découle ou la méfiance face à l'élève ou au parent et l'évitement de la confrontation), b) leur changement en pensées plus positives (plutôt que se dire «Qu'est ce que je vais encore rencontrer aujourd'hui avec cet élève ? », avec pour conséquences une anxiété face à l'élève et une agressivité, se dire « Chaque jour est différent ! Laissons-nous surprendre. ») et c) l'assouplissement des croyances (« Je dois faire face seul. »).

Grâce à l'intervention du directeur, en collaboration avec la psychologue, les enseignants ont réussi à affronter les parents présentant des comportements d'agression, en réunion de parents pour l'enseignant de $6^{\text {ème }}$ année, suite à une convocation du père agresseur par le directeur, pour l'enseignante de $3^{\text {ème }}$ année.

Les techniques qui leur ont été les plus utiles ont été celles du contrôle respiratoire et de la relaxation, utilisées avant et pendant la confrontation aux parents. Les techniques cognitives leur ont paru utiles pour changer leur point de vue face aux agresseurs et limiter les effets dépressiogènes dans le cas de l'enseignante de $3^{\text {ème }}$ année.

Les comportements d'agressions indirectes ont disparu tout de suite après la confrontation entre enseignant et parent agresseur.

Le suivi a permis de constater le maintien de contacts réguliers entre enseignants et parents (participation systématique aux réunions de parents, contacts dans la cour de 
l'école et participation à l'activité de la classe pour Mme M., lui donnant une autre occasion de contacts sociaux), tout en mettant en évidence le maintien d'une agressivité verbale directe entre Mme M. et l'enseignant de $6^{\text {ème }}$ année. De même des critiques indirectes sur les méthodes de l'enseignante de $3^{\text {ème }}$ année à la maison par M. H. ont perduré, mais pas en présence de l'enfant, aux dires de Mme H. seule interlocutrice présente pour la psychologue.

Les enseignants ont reconnu avoir continué à utiliser les techniques apprises pendant cette situation de crise et avoir accru leur sentiment de compétences personnelles pour la confrontation avec les parents. Ils souhaiteraient bénéficier à l'avenir de telles formations pour approfondir leurs connaissances sur les techniques d'affirmation de soi et les techniques cognitives.

\section{Discussion}

L'analyse comportementale permet une mise en relation claire et fonctionnelle des facteurs environnementaux et individuels responsables de comportements agressifs en milieu scolaire.

Le modèle antécédents-stimulus-comportement-conséquences, inspiré du modèle SORC parait bien adapté, en permettant de repérer les chaines de comportements qui se renforcent les uns les autres. Pour une mise en évidence plus précise des facteurs cognitifs et émotionnels individuels, le modèle SECCA [12] parait indiqué.

En cohérence avec l'analyse fonctionnelle, le plan d'intervention présenté a pris en compte des antécédents au comportement-cible pouvant jouer le rôle de facteurs causaux et des facteurs de maintien, sources de renforcement du comportement-cible. Le choix des techniques a découlé de cette analyse.

Toutefois, s'agissant d'une intervention en urgence, le nombre de séances avec la victime a été limité, ce qui a conduit à choisir les techniques les plus efficaces pour un objectif limité, soit dans les cas présentés l'arrêt de l'agression et la reprise de la communication. Par exemple, sur l'ensemble des techniques d'affirmation de soi, ont été retenus : la reconnaissance du point de vue de l'autre, l'expression émotionnelle et les repères pour faire une critique sans agressivité. En aucun cas, n'ont été traités et résolus les problèmes associés, comme le manque de compétences en gestion de soi ou en communication de l'enseignant, ce qui aurait nécessité une formation indépendante, systématique et étalée dans le temps. 
Enfin, il apparait que le contexte grec favorise l'éclosion et le maintien de telles agressions : en l'absence d'évaluation et de contrôle des compétences pédagogiques des enseignants, les parents ont développé une méfiance du système éducatif grec, qui favorise la suspicion et l'ingérence dans le fonctionnement de la classe, avec les conséquences indirectes relatées dans cet article.

En réponse à ces problèmes, il semble essentiel de développer la formation initiale et continue des enseignants à l'analyse fonctionnelle des comportements problèmes, aux techniques comportementales et cognitives appliquées aux groupes-classe [21]. En particulier, les techniques de communication affirmée, peuvent faciliter leurs relations avec les parents et avec les élèves, dans un esprit de collaboration et de partage des savoirs sur l'enfant et non de lutte de pouvoir. Il serait également utile de leur apprendre la gestion de soi [22] afin de se protéger contre le stress et de prévenir l'épuisement professionnel. Pour les chefs d'établissement, il serait souhaitable de garantir un soutien aux enseignants en situation de crise et l'aide par un psychologue spécialisé dans la gestion des ressources humaines ou des situations de stress en milieu professionnel.

En France, la réforme de la formation des enseignants, dite de la masterisation, a eu pour conséquence dans de nombreux masters «Métiers de l’Enseignement et de la Formation » des Instituts Universitaires de Formation des Maîtres (IUFM), désormais intégrés aux universités, de proposer des enseignements dédiés à la gestion de classe. Cependant, l'analyse fonctionnelle des situations de classe ou des relations individuelles n'est pas enseignée de façon systématique. Elle reste limitée aux IUFM où des formateurs ont une formation en psychologie comportementale et cognitive.

\section{Conclusion}

Les interventions cognitivo-comportementales en milieu scolaire sont pratiques, faciles à mettre en place et efficaces, surtout si elles sont associées à une analyse fonctionnelle comportementale rigoureuse. Elles répondent bien aux exigences d'intervention en situation de crise comme celle de l'agression externe d'un enseignant. Les modèles SORC sont utiles à la mise en évidence des chaines de comportements entre les différents protagonistes, enseignant, élèves, parents et équipe pédagogique. Un modèle plus complet comme une grille SECCA permet ensuite une analyse plus fine des comportements individuels. Mais ces interventions ne suffisent 
pas à régler tous les problèmes, notamment dans un contexte où les moyens de contrôle et d'aide sont si limités comme en Grèce.

\section{Conflit d'intérêt}

Aucun.

\section{Références}

[1]INSERM expertise collective. Troubles des conduites chez l'enfant et chez 1'adolescent. Paris: INSERM; 2005.

[2]Clément C, Stephan E. Favoriser les compétences sociales à l'école: un exemple de recherche-action en zone d'éducation prioritaire, Pra Psy 2006;12:447-463.

[3]Légeron P. Le stress au travail. Paris: Odile Jacob; 2003.

[4]Carra C. Violence en milieu scolaire: comment les élèves percoivent- ils les violences à l'école? NVA 2010;313:4-7.

[5]Schneider BH, Norman S, Allès-Jardel M, Provost MA, Tarabulsy G. Conduites agressives chez l'enfant: perspectives développementales et psychosociales. Québec: Presse de l'Université du Québec; 2009.

[6]Bjorkqvist K, Osterman K, Lagerspetz KMJ. Sex differences in covert aggression among adults. Aggress Behav 1994;20:27-33.

[7]Bjorkqvist K., Sex differences in physical, verbal, and indirect aggression: A review of recent research. Sex Roles 1994;30(3/4):177-188.

[8]Brunet L. Stress et climat de travail chez les enseignants. Téléchargé le 13 Mars 2011 from http://www.forres.ch/colloque/stress-climat-brunet.htm

[9]Mennuti RB, Freeman A, Christner RW. Cognitive-behavioral interventions in educational settings: A handbook for practice. New-York: Routledge Taylor \& Francis Group; 2009.

[10]Skinner, B.F., L'analyse expérimentale du comportement. Bruxelles: Dessart; 1971.

[11]Archambault J, Chouinard R. Vers une gestion éducative de la classe. Bruxelles: De Boeck; 2009.

[12]Cottraux J. Les thérapies comportementales et cognitives. Paris: Masson, 1993. 
[13]Boisvert JM, Beaudry M. S'affirmer et communiquer. Québec: Les Éditions de l'homme; 1977.

[14]Cungi C. Savoir s'affirmer en toutes circonstances. Paris: Retz; 1996.

[15]Cariou-Rognant AM, Chaperon AF, Duschene N. L'affirmation de soi par le jeu de rôle en thérapie comportementale et cognitive. Paris: Dunod; 2007.

[16] Pleux D. Exprimer sa colère sans perdre le contrôle. Paris: Odile Jacob; 2006.

[17]Van Rillaer J. Les colères. Meschers: Editions Bernet-Danilo; 1999.

[18]Servant D. Gestion du stress et de l'anxiété. Paris: Masson; 2007.

[19]Cungi C, Limousin S. Savoir se relaxer en choisissant sa méthode. Paris: Retz; 2003.

[20]Beck AT, Rush AJ, Shaw BF, Emery G. Cognitive therapy of depression. NewYork: The Guilford Press; 1979.

[21]Alberto PA, Troutman AC. Applied behavioral analysis for teachers. Upper Saddle River, New Jersey: Pearson; $8^{\text {th }}$ edition; 2009.

[22]Van Rillaer J. La gestion de soi. Sprimont: Mardaga; 1992. 